\title{
Static and Dynamic Pupil Characteristics in Myopic Anisometropic Amblyopia
}

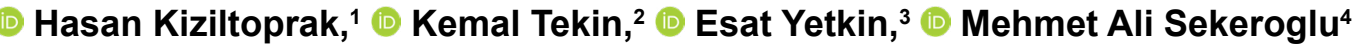 \\ 1Department of Ophthalmology, Bingol Women's Health and Children's Hospital, Bingol, Turkey \\ ${ }^{2}$ Department of Ophthalmology, Ercis State Hospital, Van, Turkey \\ ${ }^{3}$ Department of Ophthalmology, Midyat State Hospital, Mardin, Turkey \\ ${ }^{4}$ Department of Ophthalmology, Ankara Ulucanlar Eye Training and Research Hospital, Ankara, Turkey
}

\begin{abstract}
Objectives: The purpose of this study was to compare static and dynamic pupillometry measurements in patients with myopic anisometropic amblyopia with those of age-matched, healthy subjects.

Methods: This prospective, cross-sectional study consisted of 29 patients with myopic anisometropic amblyopia and 83 control subjects. While both the amblyopic eye and the fellow eye of patients with myopic anisometropic amblyopia were examined, data were only recorded for the right eye of the subjects in the control group. Static pupillometry measurements determined included the scotopic pupil diameter (PD), mesopic PD, low photopic PD, and high photopic PD. Subsequently, dynamic pupillometry measurements were obtained, including the resting diameter, amplitude of pupil contraction, latency of pupil contraction, duration of pupil contraction, velocity of pupil contraction, latency of pupil dilation, duration of pupil dilation, and velocity of pupil dilation.

Results: In the myopic anisometropia patients, there was a statistically significant difference in the low photopic PD and high photopic PD values of the amblyopic eyes and the corresponding fellow eyes compared with the healthy subjects ( $p<0.05$ for each). These parameters of low photopic PD and high photopic PD were similar between the highly myopic eyes and the fellow eyes. There was no statistically significant difference in the mesopic or scotopic PD values between any of the groups ( $p>0.05$ for each). There were no significant differences in the dynamic pupillometric measurements between the study and control eyes.

Conclusion: These results confirm that amblyopia is a binocular disorder. The dynamic pupillary responses were similar in the highly myopic and fellow eyes, indicating that evaluation of relative afferent pupillary defects may not be a useful test to differentiate amblyopic eyes from healthy corresponding eyes.

Keywords: Amblyopia, dynamic pupillometry, myopic anisometropia, static pupillometry.
\end{abstract}

\section{Introduction}

Amblyopia is defined as reduced best-corrected visual acuity (BCVA) in either one or both eyes without any organic abnormality of the eye or visual pathways (I). Amblyopia occurs secondary to an abnormal stimulus during a critical period of visual development and remains a major cause of preventable vision loss (2). This abnormal visual experience may be due to strabismus, anisometropia, high bilateral refractive errors, or visual deprivation (2). Anisometropia, in which there is a significantly different refractive power in each eye, is among the leading causes of amblyopia (3). Although there is some controversy, most authors accept an interocular difference of 1.00 diopter (D) or more as the threshold for anisometropia (3).

\section{Address for correspondence: Hasan Kiziltoprak, MD. Bingol Kadin Sagligi ve Cocuk Hastanesi, Oftalmoloji Klinigi, Bingol, Turkey}

Phone: +90 5386756528 E-mail: hsnkzltprk21@gmail.com

Submitted Date: April 11, 2020 Accepted Date: May 16, 2020 Available Online Date: July 29, 2020

${ }^{\circ}$ Copyright 2020 by Beyoglu Eye Training and Research Hospital - Available online at www.beyoglueye.com OPEN ACCESS This work is licensed under a Creative Commons Attribution-NonCommercial 4.0 International License. 
Many studies have reported that amblyopia and anisometropia were associated with developmental abnormalities of the visual system, including the visual cortex, lateral geniculate nucleus, retina, retinal nerve fiber layer, optic nerve, macula, axial length, and the cornea (4-8). The relationship between the pupil and amblyopia has been examined. Relative afferent pupillary defects (RAPDs) and impairments in the pupillary light reflexes in amblyopic eyes have been previously reported (9-12). Kocamis et al. (13) investigated pupil diameter (PD) difference in anisometropic amblyopia and demonstrated a relationship between amblyopia and anisocoria. All of these studies revealed an impaired mechanism affecting pupil physiology, which may explain the pathophysiological mechanism of amblyopia.

Developments in technology used in the assessment of pupil responses have made it possible to record quantitative, objective, noninvasive, and repeatable measurements of the PD as well as the pupillary kinetics. New, automated pupillometry devices can evaluate pupil responses statically under different illumination conditions as well as dynamically (14, 15). Although many pupil disorders have been defined in association with amblyopia, static and dynamic pupillary characteristics that could clarify the pathophysiology of amblyopia have not yet been extensively investigated. Melanopsin retinal ganglion cells, which contribute to steady-state pupil constriction, have also not been investigated in amblyopia.

The current study was designed with this perspective and the objective of using an automatic quantitative pupillometry system to investigate whether there were different static and dynamic pupil characteristics in a highly myopic eye and the fellow eye in cases of myopic anisometropia when compared with otherwise normal eyes of healthy subjects.

\section{Methods}

This prospective, cross-sectional study was performed at a tertiary ophthalmology clinic. The study protocol was approved by the Institutional Ethics Committee, and the study was performed in accordance with the ethical standards of the Declaration of Helsinki. Written, informed consent was obtained from all of the participants or their parents or legal guardians prior to enrollment.

Patients who had been diagnosed with myopic anisometropic amblyopia were enrolled in the study. Myopia was defined as $\geq-1.00 \mathrm{D}$ in the cycloplegic spherical equivalent. Myopic anisometropia was defined as myopia in both eyes and an interocular difference of $\geq 3.00 \mathrm{D}$ in the cycloplegic spherical equivalent $(7,8)$. An eye with a BCVA of $\leq 20 / 30$ or at least 2 Snellen visual acuity lines of difference in the fellow eye was considered amblyopic $(7,8)$. The mean astigmatism level was $\geq-1.00 \mathrm{D}$ in all of the groups. Both eyes of the patients with myopic anisometropic amblyopia were analyzed. The control subjects were selected from age and gender-matched patients who presented at the outpatient clinic for a routine ophthalmic examination. Only the right eye of each control subject was evaluated for the study. The highly myopic eyes of patients with myopic anisometropic amblyopia were classified as amblyopic eyes, the corresponding eyes of patients with myopic anisometropic amblyopia were classified as fellow eyes, and the right eyes of the control subjects were accepted as the control group.

Patients with strabismus, nystagmus, history of previous ocular surgery or laser treatment, trauma or uveitis, corneal disease, retinal disease, optic nerve disease and glaucoma, neurological disease or other diseases of the visual pathways, or ocular media opacities including cataract, were excluded from the study. Since smoking may be associated with changes in pupil size, only non-smokers were included $(16,17)$. Subjects were also excluded if they had used drugs or consumed alcohol during the previous year, had a diagnosis of diabetes mellitus, or had taken systemic medications during the previous 3 months. Participants with any of the following conditions that can affect pupillary motility were also excluded: iris and/or pupil anomalies such as coloboma, anisocoria, synechia, and sphincter tear; use of topical medications that may affect iris mechanics, such as tropicamide, cyclopentolate, pilocarpin, and narcotic-derived medications; neurological disease or other diseases of the visual pathways; and those who did not elect to undergo pupillometry examinations.

A full ophthalmic examination, including BCVA testing with a Snellen chart, intraocular pressure measurement, slit-lamp biomicroscopy, and a dilated fundus examination was performed for all of the study participants. Three drops of cyclopentolate hydrochloride $1 \%$ were administered at 5-minute intervals to induce pupil dilation, and autorefraction was performed using a single automatic refractor-keratometer device (RF-K2 full auto ref-keratometer; Canon, Tokyo, Japan) approximately 45 minutes after the last drop in each case. The mean of 3 successful measurements was taken for analysis. The refractive error was calculated using the cycloplegic spherical equivalent ( $\mathrm{SE}=$ spherical component + I/2 cylinder component).

The pupillometry measurements were performed by the same clinician using an automatic quantitative pupillometry device (MonPackONE vision monitoring system; Metrovision, Perenchies, France). Contact ocular examination and pupil dilatation were avoided prior to the pupillometry examination. Pupillometry measurements were determined at least 3 days after the cycloplegic measurements. The quantitative pupillometry system was equipped with near-infrared illumination and a high-resolution camera $(880 \mathrm{~nm})$, which allowed for measurements to be obtained from binocular pupils in complete darkness and precise control of stimula- 
tion parameters. A white stimulus was used, obtained from a full-field backlight that combined red $(632 \mathrm{~nm})$, green (523 $\mathrm{nm})$, and blue $(465 \mathrm{~nm})$ light-emitting diode sources. This system allowed the clinician to record both static and dynamic pupillometry measurements and to perform accurate measurement of pupil size (accuracy $=0.1 \mathrm{~mm}$ ) (14). Three consecutive measurements of each subject were obtained and the average values were selected for data analysis. To minimize examiner-induced errors, the automatic-release mode of the device was used and only high-quality images were included in the study. The pupillometry measurements were performed at the same time of day (10:00 am-12:00 $\mathrm{pm}$ ) and in the same environmental conditions (uniform lighting conditions) in order to minimize the effect of circadian variation on pupillary response (18). The participants were required to fixate on a target in the center of the test field while the stimuli were presented in order to control fixation stability during pupil recording. The pupil recordings were only used in the study analysis if eye movements were within $5^{\circ}$ of the central fixation axis of the optical system and the infrared camera plane (19). The pupil contours were outlined on the image during the measurements to allow for control of measurement accuracy and analysis. The proprietary analysis software of the MonPackONE device was used to conduct automatic static and dynamic pupillometry. The pupillary contours on the images were automatically outlined by the software, ensuring that measurements were accurate and recorded under controlled lighting conditions (Fig. I). Subsequently, the software performed an analysis of the temporal and the average response to successive visual stimuli with automated quantification of the following parameters: latency and duration of contraction and dilatation (ms); initial, minimum, maximum, and mean PD (mm); am- plitude of contraction $(\mathrm{mm})$; and contraction and dilatation speed (velocity) of the pupil (mm/s) (Fig. I).

Static pupillometry was measured using several levels of illumination to measure pupil size in the following vision conditions: scotopic $\left(0.1 \mathrm{~cd} / \mathrm{m}^{2}\right)$, mesopic $\left(1 \mathrm{~cd} / \mathrm{m}^{2}\right)$, low photopic $\left(10 \mathrm{~cd} / \mathrm{m}^{2}\right)$, and high photopic $\left(100 \mathrm{~cd} / \mathrm{m}^{2}\right)$. Scotopic PD, mesopic PD, low photopic PD, and high photopic PD values were recorded (Fig. I). Dynamic pupillometry measurements were obtained in darkness in a period of 90 seconds after 5 minutes of adaptation to the darkness. The participants were examined using white light flashes (stimulation on time: $200 \mathrm{~ms}$, stimulation off time: $3300 \mathrm{~ms}$; total luminance: $100 \mathrm{~cd} / \mathrm{m}^{2}$; total intensity 20 lux). The stimuli were full-field (ganzfeld), seen at a distance of $30 \mathrm{~cm}$. Images of both eyes were acquired and processed in real time (30 images per second). A Minolta LSI 00 luminance meter (Konika Minolta, Tokyo, Japan) was used to measure luminance output. The average response to successive visual stimuli (light flashes) was quantified using the following parameters: resting diameter, amplitude of pupil contraction, latency of pupil contraction, duration of pupil contraction, velocity of pupil contraction, latency of pupil dilation, duration of pupil dilation, and velocity of pupil dilation (Fig. I).

\section{Statistical Analysis}

The data obtained were analyzed using IBM SPSS Statistics for Windows, Version 22.0 software (IBM Corp., Armonk, NY, USA). Descriptive statistics are presented as mean \pm SD, frequency distributions, and percentages. A chi-square test was used to analyze categorical variables. Normal distribution was tested using analytical methods (Kolmogorov-Smirnov/Shapiro-Wilk tests). To compare the amblyopic eyes with the fellow eyes, a paired samples t-test was used
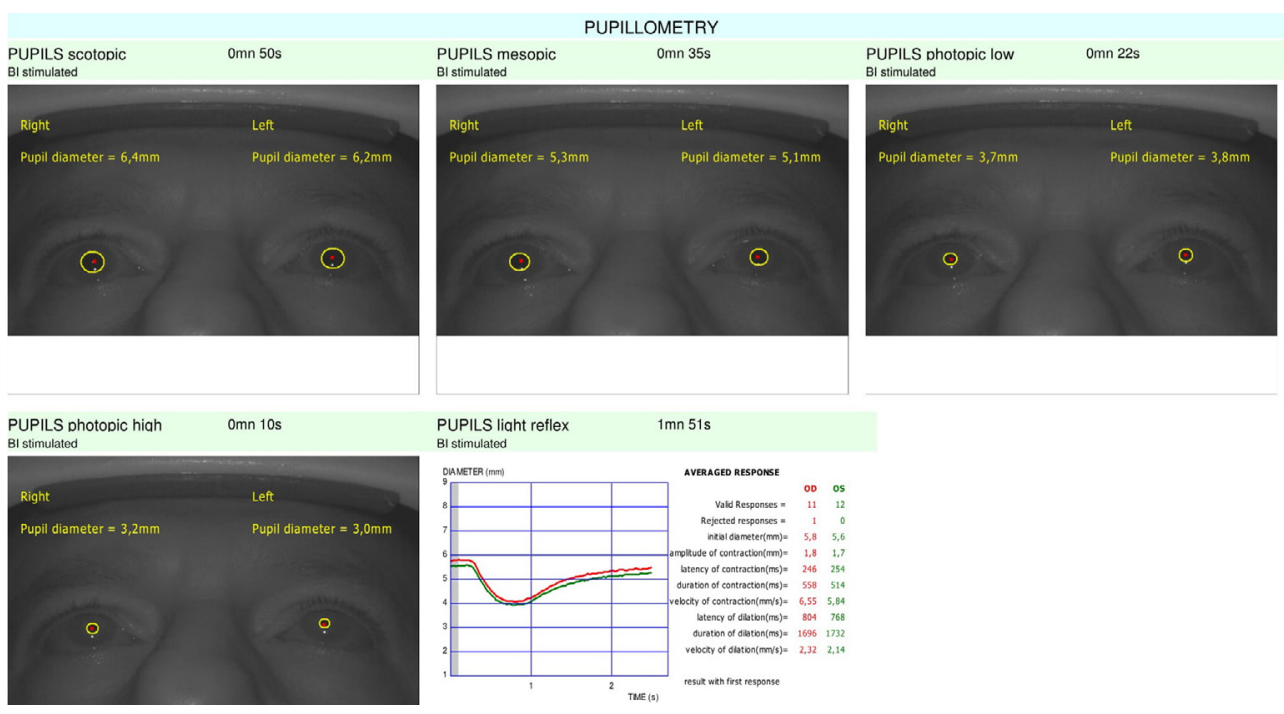

Figure I. An example of static and dynamic pupillary characteristics determined using an automatic, quantitative, pupillary measurement system. BI: Base-in. 
for normally distributed variables and the Wilcoxon signedrank test was used for variables that did not show normal distribution. To compare the amblyopic eyes with the control eyes, as well as the fellow eyes with the control eyes, an independent sample t-test was used for normally distributed data and the Mann-Whitney $U$ test for those that were not non-normally distributed. A probability level of $p<0.05$ was considered statistically significant.

\section{Results}

This study analysis included 29 patients who had myopic anisometropic amblyopia ( 13 males, 16 females) with a mean age of $21.4 \pm 9.8$ years and 83 control subjects ( 35 males, 48 females) with a mean age of $20.0 \pm 10.2$ years. There was no significant difference between the groups in terms of age or gender ( $p>0.05$ for each; Table I). The mean BCVA in the amblyopic eyes was statistically significantly worse than that of the fellow and control eyes $(p<0.00 \mathrm{I})$. The mean refractive error was statistically significantly greater in the amblyopic eyes compared with the fellow and control eyes $(p<0.00 \mathrm{I})$. The demographic data and clinical characteristics of the groups are presented in Table I.

As illustrated in Table 2, all of the static pupillometry measurements were lowest in the control eyes and highest in the fellow eyes. The amblyopic eyes and both eyes of those patients had statistically significantly greater low photopic $\mathrm{PD}$ and high photopic PD values compared with the control eyes ( $p<0.05$ for each). These parameters (low photopic PD and high photopic PD) were similar in the amblyopic and fellow eyes. There were no statistically significant differences between any of the groups in the mesopic and scotopic PD values ( $p>0.05$ for each).

Table I. Demographics and clinical characteristics of the study groups

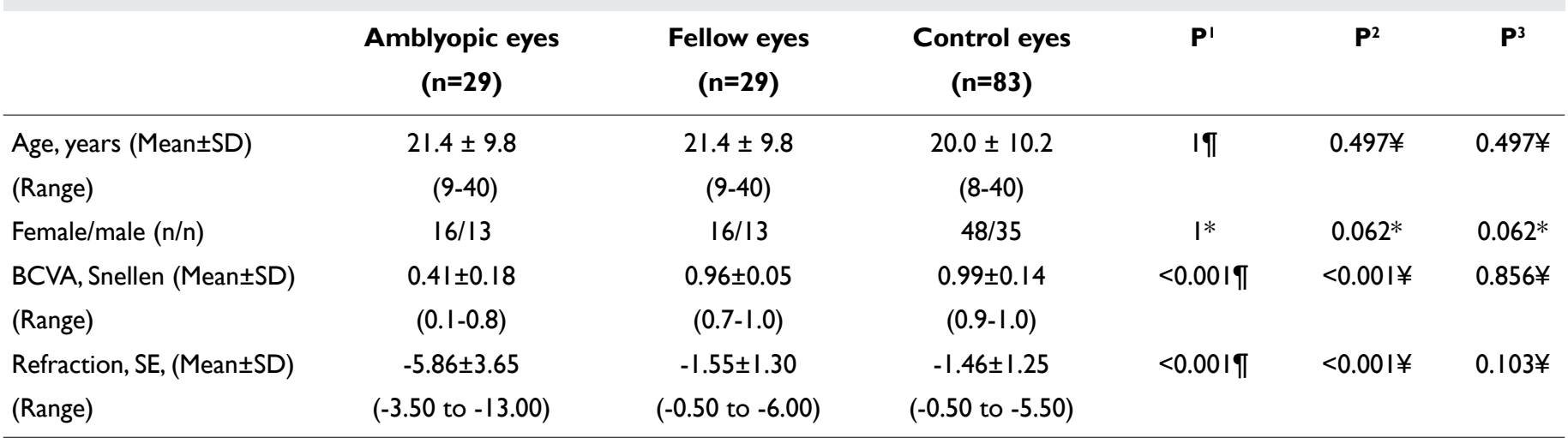

* Chi-square test; 'Difference between amblyopic eyes and fellow eyes: TPaired samples t-test; ${ }^{2}$ Difference between amblyopic eyes and control eyes: $¥$ Independent samples t-test; ${ }^{3}$ Difference between fellow eyes and control eyes: ¥Independent samples t-test; Bold values indicate $p<0.05$; BCVA: Best corrected visual acuity; SE: Spherical equivalent.

Table 2. Static pupillometry values of the study groups

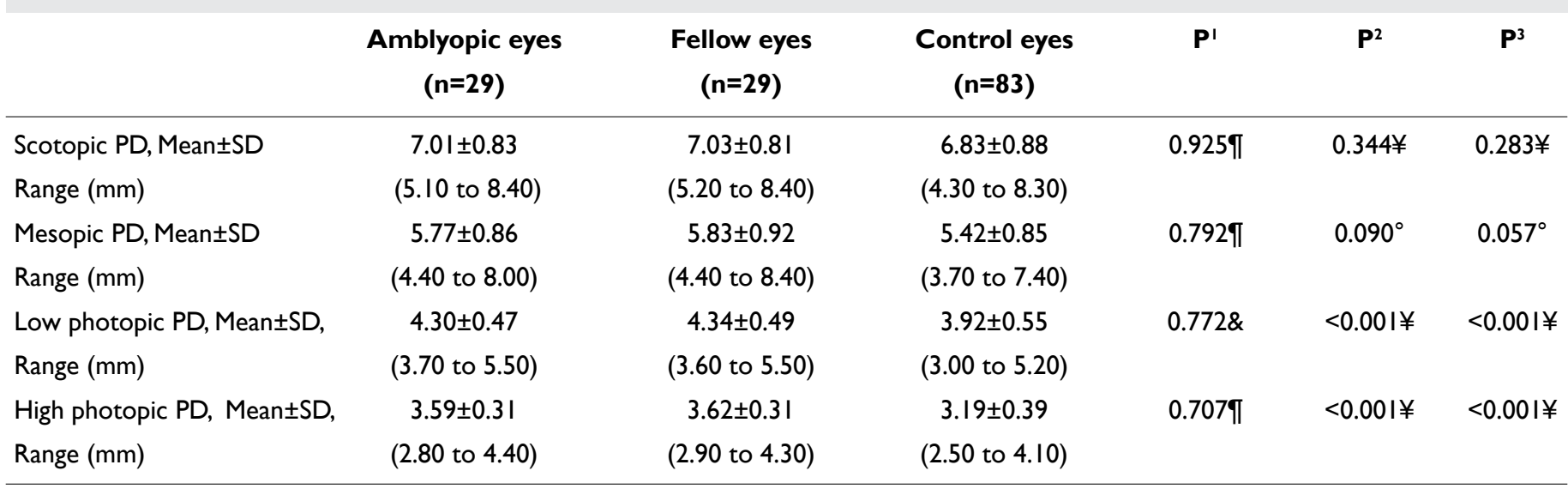

*Chi-square test. 'Differences between amblyopic eyes and fellow eyes: \Paired samples t-test, \&Wilcoxon signed rank test; ${ }^{2}$ Differences between amblyopic eyes and control eyes: $¥$ Independent samples t-test, ${ }^{\circ}$ Mann-Whitney $U$ test; ${ }^{3}$ Differences between fellow eyes and control eyes: $¥$ Independent samples $t$-tests, ${ }^{\circ}$ Mann-Whitney $U$ test. Bold values indicate $p<0.05$. PD: Pupil diameter. 
The dynamic pupillometry measurements of the groups are shown in Table 3. There were no significant differences between the study and control eyes with respect to resting diameter, amplitude of pupil contraction, latency of pupil contraction, duration of pupil contraction, velocity of pupil contraction, latency of pupil dilation, duration of pupil dilation, and velocity of pupil dilation values ( $p>0.05$ for each).

\section{Discussion}

An automatic quantitative pupillometry system was used in this study to evaluate the static and dynamic pupillary parameters of patients with myopic anisometropic amblyopia and healthy control subjects. The objective was to determine whether or not there was a relationship between pupil size and myopic anisometropic amblyopia.

Pupillary examinations may help in the diagnosis of many ocular and neurological disorders. However, subjective analysis of pupillary responses can be difficult due to differences in lighting conditions, changes in the intensity of light stimulus, and the clinician's experience. Many devices, such as the Pentacam (Oculus, Arlington, WA, USA) which has poor repeatability in pupil measurements, and an ocular wavefront analyzer, which is not useful under different illumination conditions, have been used for pupillary examinations $(13,20)$.
These shortcomings limit their utility. New developments in pupillometry devices have enabled the ability to perform automatic, multiple, and repeatable pupillary measurements under different illumination conditions both statically and dynamically, and the validity of results has been demonstrated to be reliable $(14,15)$.

The results of this study showed that the amblyopic eyes and the fellow eyes of patients with myopic anisometropic amblyopia had significantly greater high photopic and low photopic PD values compared with the control subjects. Kocamis et al. (13) evaluated PD differences in an analysis of anisometric ambliyopic and fellow eyes using an ocular wavefront analyzer and found significantly different PD values between amblyopic and fellow eyes. However, there were no measurements recorded in different illumination conditions. The importance of the appropriate pattern, brightness, and contrast to stimulate the cortex to activate the pupil response in order to detect pupil defects in amblyopic patients has previously been reported (2I). Therefore, an automatic pupillometry system equipped for measurements in different lighting conditions was used in this study, which eliminated some environmental factors that affect pupil responses, and more detailed and reliable results were obtained. The differences in low and high photopic PD when compared with the

Table 3. Dynamic pupillometry values of the study groups

\begin{tabular}{|c|c|c|c|c|c|c|}
\hline & $\begin{array}{l}\text { Amblyopic eyes } \\
\qquad(n=29)\end{array}$ & $\begin{array}{l}\text { Fellow eyes } \\
\qquad(n=29)\end{array}$ & $\begin{array}{l}\text { Control eyes } \\
\qquad(n=83)\end{array}$ & $\mathbf{P}^{\prime}$ & $\mathbf{P}^{2}$ & $\mathbf{P}^{3}$ \\
\hline Resting diameter, Mean $\pm S D$ & $6.35 \pm 0.64$ & $6.43 \pm 0.72$ & $6.11 \pm 0.80$ & $0.548 \pi$ & 0.1567 & $0.060 ¥$ \\
\hline Range (mm) & $(5.20-7.30)$ & $(5.10-7.70)$ & $(4.50-7.60)$ & & & \\
\hline Amplitude of PC, Mean \pm SD & $|.88 \pm 0.3|$ & $1.98 \pm 0.35$ & $1.91 \pm 0.26$ & 0.2619 & $0.639 ¥$ & $0.258 ¥$ \\
\hline Range (mm) & $(1.00-2.50)$ & $(1.00-2.60)$ & $(1.30-2.50)$ & & & \\
\hline Latency of PC, Mean \pm SD & $252.6 \pm 81.1$ & $251.4 \pm 70.3$ & $260.7 \pm 69.7$ & $0.876 \&$ & $0.416^{\circ}$ & $0.455^{\circ}$ \\
\hline Range (ms) & $(30-310)$ & $(28-304)$ & $(36-3 \mid 4)$ & & & \\
\hline Duration of PC, Mean \pm SD & $633.7 \pm 80.2$ & $663.9 \pm 99.2$ & $624.9 \pm 68.2$ & $0.208 \rrbracket$ & $0.58 I ¥$ & 0.1097 \\
\hline Range, (ms) & $(450-928)$ & $(448-I I 6 I)$ & $(473-869)$ & & & \\
\hline Velocity of PC, Mean \pm SD & $6.03 \pm 1.15$ & $6.44 \pm 1.24$ & $6.08 \pm 0.86$ & $0.201 \pi$ & $0.808 ¥$ & $0.092 ¥$ \\
\hline Range, $(\mathrm{mm} / \mathrm{s})$ & $(3.18-8.32)$ & $(3.91-9.72)$ & $(4.15-7.97)$ & & & \\
\hline Latency of PDil, Mean \pm SD & $870.2 \pm 66.8$ & $885.3 \pm 89.3$ & $855.6 \pm 59.6$ & 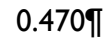 & $0.275 ¥$ & $0.105 ¥$ \\
\hline Range (ms) & $(740-1032)$ & $(73 \mid-1168)$ & $(736-1000)$ & & & \\
\hline Duration of PDil, Mean \pm SD & I 583.4 \pm 85.4 & $1555.8 \pm 1 \mid 7.2$ & I 593.3 \pm 82.3 & $0.361 \pi$ & $0.195 ¥$ & $0.069 ¥$ \\
\hline Range (ms) & $(1300-1705)$ & $(\mid 201-1737)$ & $(1136-1763)$ & & & \\
\hline Velocity of PDil, Mean \pm SD & $2.81 \pm 1.89$ & $2.87 \pm 1.70$ & $2.62 \pm 1.87$ & $0.79 \mid \&$ & $0.448^{\circ}$ & $0.163^{\circ}$ \\
\hline Range (mm/s) & $(1.35-8.11)$ & $(1.60-7.75)$ & $(1.85-8.03)$ & & & \\
\hline
\end{tabular}

'Differences between amblyopic eyes and fellow eyes: \Paired samples t-test, \&Wilcoxon signed-rank test; ${ }^{2}$ Differences between amblyopic eyes and control eyes: ¥Independent samples t-test, ${ }^{\circ}$ Mann-Whitney $U$ test; ${ }^{3}$ Differences between fellow eyes and control eyes: $¥$ Independent samples $t$-test, ${ }^{\circ}$ Mann-Whitney $U$ test; Bold values indicate $p<0.05$. PC: Pupil contraction; PDil: Pupil dilation. 
control eyes revealed an impaired mechanism affecting pupil physiology. No differences in mesopic and scotopic conditions were detected. This could be explained by abnormal melanopsin function, which is associated with post-illumination pupil response (PIPR). Intrinsically photosensitive retinal ganglion cells are the predominant source of innervation of the olivary pretectal nucleus shell for pupil control. These inner retinal photoreceptors entirely drive the PIPR, which established markers of direct, intrinsic melanopsin activity, and sustained pupilloconstriction after light offset. It may be that melanopsin function is affected in amblyopic patients.

There is still disagreement regarding the pathophysiology of amblyopia. Some studies have revealed evidence suggesting that the major problem is at the primary visual cortex (22). However, some researchers have reported an exact pathology related to the lateral geniculate nucleus $(23,24)$. A relationship between amblyopia and neurotransmitters, such as catecholamines, glutamate, acetylcholine, cyclic adenosine monophosphate, gamma-aminobutyric acid, and serotonin, has been reported (25). In addition, these above-mentioned neurotransmitters play an essential role in the parasympathetic and sympathetic autonomic nervous systems. Thus, it can be hypothesized that impairments in the autonomic nervous system may be the reason for different PD values when compared with healthy control subjects. This also suggests that amblyopia is a binocular condition and that impairments in the cortex can cause bilateral involvement, resulting in large high photopic and low photopic PD measurements when compared with control eyes.

Many ocular conditions may affect pupil size. It has been reported that refractive error differences may affect PD. Cakmak et al. (26) found that myopic eyes had a larger mesopic PD, and found that astigmatism had a significant effect on mesopic PD. In the current study, no significant difference was detected between eyes with a high degree of myopia and the fellow eyes of cases with myopic anisometropic amblyopia compared with control eyes. With respect to the findings of the Cakmak study, the findings may have been the result of refractive differences between the eyes. There might be a smaller mesopic PD in highly myopic eyes, but refractive error differences might equalize these differences. As expected, no difference was detected between the fellow eyes of the highly myopic eyes and the control subjects, with a similar refractive error determined in both groups. The astigmatism parameters of all of the groups were similar, excluding any effect of astigmatism on PD.

Dynamic responses of the pupil are also important in the evaluation of pupillary responses in amblyopia. In the current study, no differences were detected in the dynamic responses in the 3 study groups. RAPD evaluation is a valuable diagnostic test to distinguish several ocular pathologies; therefore, it may be useful for earlier detection of amblyopia. However, it is known that amblyopic eyes may have a fairly small RAPD $(\leq 0.6$ logMAR units), which makes it difficult to distinguish $(9,20)$. A recent study conducted by Bitirgen et al. (27) investigated the dynamic pupillary responses of patients with anisometropic amblyopia and reported that these eyes had a shorter contraction duration when compared with the fellow eyes. They stated that the shorter contraction time may not only facilitate early diagnosis of amblyopia, but may also be useful to help explain mechanisms involved in amblyopia pathophysiology (27). Kase et al. (28) found no significant alteration in the amplitude or maximum velocity of the contraction in amblyopic and normal eyes using an infrared electropupillogram. A significantly longer latency of contraction was also detected when the amblyopic eyes were stimulated than when the normal eyes were stimulated. Similarly, Donahue et al. (29) investigated the contraction amplitude of pupil responses using automated pupil perimetry and found that amblyopia produced a global depression of focal pupillary responses across the entire $30^{\circ}$ field. In contrast, Miki et al. (30) also examined contraction amplitude of the pupil in amblyopic eyes and did not find any significant difference between even densely amblyopic eyes and their fellow eyes. Likewise, in the current study, no significant differences in the dynamic pupillary response were observed when the 3 groups were compared. Thus, as the dynamic response in the amblyopic eyes and fellow eyes were not significantly different, RAPD assessment may not be the most useful tool to detect anisometropic amblyopia earlier in these cases. However, the pupil light response is affected in the fellow eye of amblyopic individuals where there is normal visual acuity, so it is a more sensitive measure of retinal and optic nerve function in nonamblyopic fellow eyes compared with visual acuity.

This study has some limitations. First, the number of patients was relatively small, which could affect the validity of the results. Second, PD differences may be observed in subjects without any abnormal or pathological condition, known as physiological anisocoria, and this is another factor that could have affected the results (3I). Third, the fixation target of the pupillometry system used could have resulted in adaptation and bias the baseline PD toward the smaller side in all our measurements. Finally, the generalizability of the findings might be limited as a result of the cross-sectional design of the study.

\section{Conclusion}

The results of this study confirmed that amblyopia is a binocular disorder. It was observed that the static pupil characteristics of highly myopic eyes and their fellow eyes in myopic anisometropia were similar, and that the high photopic PD, and low photopic PD values were different from those of 
healthy subjects. As the dynamic pupillary responses were similar in the highly myopic eyes and their fellow eyes, RAPD testing may not be the most useful means to differentiate amblyopic eyes from healthy fellow eyes.

\section{Disclosures}

Ethics Committee Approval: Ankara Numune Training and Research Hospital Ethics Committee E-I7-1542/27.09.2017.

Peer-review: Externally peer-reviewed.

Conflict of Interest: None declared.

Authorship Contributions: Involved in design and conduct of the study (HK, KT, MAŞ); preparation and review of the study (KT, EY, MAŞ); data collection (HK, EY); and statistical analysis (HK, KT).

\section{References}

I. de Zárate BR, Tejedor J. Current concepts in the management of amblyopia. Clin Ophthalmol 2007; I:403-I4.

2. Simons K. Amblyopia characterization, treatment, and prophylaxis. Surv Ophthalmol. 2005;50:123-66. [CrossRef]

3. Vincent SJ, Collins MJ, Read SA, Carney LG. Myopic anisometropia: ocular characteristics and aetiological considerations. Clin Exp Optom 2014;97:29I-307. [CrossRef]

4. Patel VS, Simon JW, Schultze RL. Anisometropic amblyopia: axial length versus corneal curvature in children with severe refractive imbalance. J AAPOS 2010;|4:396-8. [CrossRef]

5. Li Q, Jiang Q, Guo M, Li Q, Cai C, Yin X. Grey and white matter changes in children with monocular amblyopia: voxel-based morphometry and diffusion tensor imaging study. $\mathrm{Br} \mathrm{J} O$ Phthalmol 2013;97:524-9. [CrossRef]

6. Andalib D, Javadzadeh A, Nabai R, Amizadeh Y. Macular and retinal nerve fiber layer thickness in unilateral anisometropic or strabismic amblyopia. J Pediatr Ophthalmol Strabismus 2013;50:218-2I. [CrossRef]

7. Pang Y, Allen M, Robinson J, Frantz KA. Contrast sensitivity of amblyopic eyes in children with myopic anisometropia. Clin Exp Optom 2019;102:57-62. [CrossRef]

8. Pang Y, Allison C, Frantz KA, Block S, Goodfellow GW. A prospective pilot study of treatment outcomes for amblyopia associated with myopic anisometropia. Arch Ophthalmol 20I 2; I30:579-84. [CrossRef]

9. Portnoy JZ, Thompson HS, Lennarson L, Corbett JJ. Pupillary defects in amblyopia. Am J Ophthalmol 1983;96:609-14.

10. Feng Y, Li W, Liu Y. Macular light sensitivity and afferent pupillary function in children's amblyopia. [Article in Chinese] Zhonghua Yan Ke Za Zhi 1997;33: I I3-6.

II. Firth AY. Pupillary responses in amblyopia. $\mathrm{Br} J$ Ophthalmol 1990;74:676-80. [CrossRef]

12. Barbur JL, Hess RF, Pinney HD. Pupillary function in human amblyopia. Ophthalmic Physiol Opt 1994; I4:I39-49. [CrossRef]

13. Kocamıs SI, Cakmak HB, Cagıl N. Investigation of the pupil diameter differences in anisometropic amblyopia. Turk J Ophthalmol 20l3;43:45-50. [CrossRef]
14. Tekin K, Sekeroglu MA, Kiziltoprak H, Doguizi S, Inanc M, Yilmazbas P. Static and dynamic pupillometry data of healthy individuals. Clin Exp Optom 2018;101:659-65. [CrossRef]

I5. Bootsma S, Tahzib N, Eggink F, de Brabander J, Nuijts R. Comparison of two pupillometers in determining pupil size for refractive surgery. Acta Ophthalmol Scand 2007;85:324-8. [CrossRef]

16. Lie TC, Domino EF. Effects of tobacco smoking on the human pupil. Int J Clin Pharmacol Ther 1999;37:184-8.

17. Erdem U, Gundogan FC, Dinc UA, Yolcu U, Ilhan A, Altun S. Acute effect of cigarette smoking on pupil size and ocular aberrations: a pre- and postsmoking study. J Ophthalmol 20। 5;20। 5:625470. [CrossRef]

18. Zele AJ, Feigl B, Smith SS, Markwell EL. The circadian response of intrinsically photosensitive retinal ganglion cells. PLoS One 201 1;6:el 7860. [CrossRef]

19. Adhikari P, Zele AJ, Feigl B. The Post-Illumination Pupil Response (PIPR). Invest Ophthalmol Vis Sci. 20I5;56:3838-49.

20. Shankar H, Taranath D, Santhirathelagan CT, Pesudovs K. Anterior segment biometry with the Pentacam: comprehensive assessment of repeatability of automated measurements. J Cataract Refract Surg 2008;34:103-13. [CrossRef]

21. Law CL, Siu M, Modica P, Backus B. Stimulus characteristics affect assessment of pupil defects in amblyopia. Optom Vis Sci 20I5;92:55I-8. [CrossRef]

22. Conner IP, Odom JV, Schwartz TL, Mendola JD. Monocular activation of VI and V2 in amblyopic adults measured with functional magnetic resonance imaging. J AAPOS 2007; I I:34I-50.

23. Miki A, Liu GT, Goldsmith ZG, Liu CS, Haselgrove JC. Decreased activation of the lateral geniculate nucleus in a patient with anisometropic amblyopia demonstrated by functional magnetic resonance imaging. Ophthalmologica 2003;217:365-9.

24. Lempert P. Retinal area and optic disc rim area in amblyopic, fellow, and normal hyperopic eyes: a hypothesis for decreased acuity in amblyopia. Ophthalmology 2008; I 15:2259-6I. [CrossRef]

25. Bear MF, Singer W. Modulation of visual cortical plasticity by acetylcholine and noradrenaline. Nature 1986;320:172-6.

26. Cakmak HB, Cagil N, Simavli H, Duzen B, Simsek S. Refractive error may influence mesopic pupil size. Curr Eye Res 2010;35:130-6. [CrossRef]

27. Bitirgen G, Daraghma M, Özkağnıcı A. Evaluation of Pupillary Light Reflex in Amblyopic Eyes Using Dynamic Pupillometry. Turk J Ophthalmol 2019;49:310-4. [CrossRef]

28. Kase M, Nagata R, Yoshida A, Hanada I. Pupillary light reflex in amblyopia. Invest Ophthalmol Vis Sci 1984;25:467-7I.

29. Donahue SP, Moore P, Kardon RH. Automated pupil perimetry in amblyopia: generalized depression in the involved eye. Ophthalmology 1997; 104:2 I6I-7. [CrossRef]

30. Miki A, lijima A, Takagi M, Yaoeda K, Usui T, Hasegawa S, et al. Pupillography of automated swinging flashlight test in amblyopia. Clin Ophthalmol 2008;2:78I-6. [CrossRef]

31. Rosenberg ML. Physiologic Anisocoria: A manifestation of a physiologic sympathetic asymmetry. Neuro-Ophthalmology 2008;32: |47-9. [CrossRef] 